\title{
Estimation du stock de carbone organique dans les plantations de Acacia auriculiformis A. Cunn. ex Benth. des forêts classées de Pahou et de Ouèdo au Sud du Bénin
}

\author{
Gomido Xavier KOOKE ${ }^{1 *}$, Rachad Kolawolé Foumilayo Mandus ALI ${ }^{1}$, \\ Jean-Marie DJOSSOU ${ }^{1}$ et Ismaïla TOKO IMOROU ${ }^{2}$ \\ ${ }^{1}$ Laboratoire de Biogéographie et Expertise Environnementale, Département de Géographie et Aménagement \\ du Territoire, Faculté des Sciences Humaines et Sociales, Université d'Abomey-Calavi, Bénin. \\ ${ }^{2}$ Laboratoire de Cartographie, Département de Géographie et Aménagement du Territoire, Faculté des \\ Sciences Humaines et Sociales, Université d'Abomey-Calavi, Bénin. \\ *Auteur correspondant, E-mail : gkooke@gmail.com
}

\section{RESUME}

La réduction du dioxyde de carbone par les arbres est une importante activité écosystémique. La présente étude vise à déterminer le stock de carbone organique dans les plantations de Acacia auriculiformis des forêts classées (Ouèdo et Pahou) afin d'appréhender leur contribution à l'atténuation des changements climatiques. La méthodologie d'estimation du carbone est basée sur les recommandations faites par l'IPCC et celle de FAO ainsi que des équations allométriques. Les données climatiques ont été collectées à la station météorologique d'Agonkanmè. Selon les différents compartiments, les résultats révèlent que le stock de carbone est plus élevé dans le tronc : 78,17\%; les branches : 19,50\% et les feuilles: $2,33 \%$. Le potentiel de séquestration du carbone du sol varie entre $31,46 \%$ et $70,99 \%$ en fonction des types de sol. Les stocks de carbone élevés sont obtenus dans la plantation de 2,5 ans sur sol hydromorphe à Pahou $(106,23 \pm 38,39$ tC/ha) contre $77,77 \pm 5,47 \mathrm{tC} /$ ha pour celle de 2 ans sur sol ferralitique à Ouèdo. Il y a une corrélation fortement négative et significative $(\mathrm{r}=-0,931, \mathrm{P}<0,001)$ entre la température et le stock de carbone. Les fortes températures observées sont le résultat de la faible séquestration de carbone et vice-versa.

(C) 2019 International Formulae Group. All rights reserved.

Mots clés: Séquestration de carbone, essences exotiques, atténuation des changements climatiques, Benin

\section{Estimation of the organic carbon's stock in the plantations of Acacia auriculiformis A. Cunn. ex Benth. of the classified forests of Pahou and Ouedo in the South of Benin}

\begin{abstract}
The reduction by trees of carbon dioxide is an important ecosystem activity. The present study aimed at assessing the organic carbon stock in the plantations of Acacia auriculiformis of the classified forests of
\end{abstract}


Ouèdo and Pahou in order to better understanding their contribution to mitigating climate changes. The method described by IPCC (2007), FAO (2002) associated with allometrics equations were used for the carbon stock estimation. The climate datas used, were collected at the Meteorological Station of Agonkanmè. According to the different components of trees, results shown that the trunk has the highest rate of carbon stock: $78.17 \%$; branches: $19.50 \%$ and leaves: $2.33 \%$. The potential of soil carbon's sequestration varies between $31.46 \%$ and $70.99 \%$ depending on soil types. The high-level stocks of carbon are obtained in 2.5 years old on a hydromorphic soil plantation in Pahou (106.23 $\pm 38.39 \mathrm{tC} / \mathrm{ha})$ compared with $77.77 \pm 5.47 \mathrm{tC} /$ ha from the 2 years old plantation on a ferralitic soil in Ouèdo. There is a high negative and significant correlation $(\mathrm{r}=-$ $0.931, \mathrm{P}<0.001$ ) between temperature and carbon's stock. The high temperatures observed are the result of low carbon's sequestration.

(C) 2019 International Formulae Group. All rights reserved.

Keywords: Carbon sequestration, exotic species, climate change mitigation, Benin.

\section{INTRODUCTION}

Ces dernières années, la recherche sur le changement climatique a avancé de manière remarquable (Durot, 2013). Certaines de ces recherches confirment que les émissions de gaz à effet de serre (GES) provenant des activités humaines, telles que l'industrialisation, le changement d'utilisation de terres et l'exploitation démesurée des ressources naturelles sont responsables du réchauffement climatique que l'on observe actuellement sur Terre ( Saïdou et al., 2012). En général, le changement climatique a un impact sur l'environnement dans lequel vivent les humains, affectant ainsi les moyens de subsistance de nombreuses personnes et les revenus des pays (GIEC, 2013). En effet, le $\mathrm{CO} 2$ est un gaz qui permet à la terre de bénéficier d'un effet de serre naturel indispensable à la vie, puisqu'il maintient la température moyenne terrestre à $15^{\circ} \mathrm{C}$ au lieu de $-18{ }^{\circ} \mathrm{C}$. Par ailleurs, l'augmentation de sa concentration a une influence directe sur l'efficacité de cet effet de serre en induisant le réchauffement de la planète et le changement climatique (Muoghalu, 2014). Ce dérèglement du climat est responsable de nombreuses catastrophes qui grossissent d'années en années le nombre de victimes en situation d'urgence humanitaire. Bien que les impacts négatifs associés aux émissions de $\mathrm{CO} 2$ soient clairement reconnus et identifiés, il est fort probable que dans les prochaines décennies, ces émissions se poursuivent, voire s'intensifient (Mbow, 2014). Dans le bilan global de carbone, les écosystèmes terrestres sont reconnus comme jouant un rôle essentiel, absorbant près de $30 \%$ du $\mathrm{CO} 2$ anthropique total émis (GIEC, 2013). Ayant pris conscience de cette réalité, la communauté internationale a placé le changement climatique au cœur de ses préoccupations. C'est dans cette optique qu'à la conférence des parties de la Convention Cadre des Nations Unies pour les Changements Climatiques (CCNUCC), la conférence des parties (CoP21) à Paris en 2015 sur le climat a réunis 195 pays pour décider des mesures à mettre en place, dans le but de limiter le réchauffement climatique (Pascal et Peter, 2015). Un accord international sur le climat, applicable à tous les pays, est validé par tous les participants, fixant comme objectif une limitation du réchauffement mondial entre 1,5 ${ }^{\circ} \mathrm{C}$ et $2{ }^{\circ} \mathrm{C}$ d'ici 2100 (Frédérick, 2015). A cet effet, l'accord, censé entrer en vigueur en 2020, devra à la fois traiter de l'atténuation (la baisse des émissions de gaz à effet de serre) et de l'adaptation des sociétés aux dérèglements climatiques existants et à venir (Yameogo et al., 2013). En d'autres termes, les forêts ainsi que les plantations constituent une composante importante dans le changement 
global parce qu'elles peuvent présenter, selon leur mode de gestion, un impact positif ou négatif du changement climatique induit par l'homme (Ngueguim et al., 2015 ; Bello et al., 2017). En effet, il est observé une grande capacité de stockage de $\mathrm{C}$ due à l'absorption du $\mathrm{CO} 2$ de l'atmosphère pour une forêt en croissance. Au Sud-Bénin comme dans la plupart des pays, les ressources forestières, déjà peu disponibles pour bon nombre de communautés, sont menacées par plusieurs problèmes environnementaux. Ainsi, la question de l'environnement est marquée par des déboisements anarchiques et incontrôlés résultant en partie de la pratique de l'agriculture itinérante sur brûlis (Vroh et al., 2014). L'option des plantations d'essences exotiques à croissance rapide et à rendement élevé en produits ligneux a été adoptée surtout au sud du Bénin (Djègo, 2006). Acacia auriculiformis, originaire du Nord de l'Australie, de Papouasie Nouvelle-Guinée et d'Indonésie, est introduite dans les plantations domaniales des forêts classées de Pahou et de Ouèdo. Cette conversion de forêts naturelles en plantations forestières exotiques impliquerait le remplacement d'un milieu à biodiversité stable et riche par une forêt artificielle presque mono-spécifique (Djègo et Sinsin, 2006). Ce système forestier constituerait une importe source de séquestration de C. Ainsi, l'objectif de ce travail consiste à évaluer le stock de carbone organique emmagasiné dans les plantations de Acacia auriculiformis de Pahou et Ouèdo au Sud du Bénin pour une atténuation des effets des changements climatiques au Bénin.

\section{MATERIEL ET METHODES Milieu d'étude}

Situées au Sud du Bénin, les forêts classées de Pahou et de Ouèdo se trouvent respectivement dans les communes de Ouidah et de Abomey-Calavi (département de l'Atlantique). Géographiquement, la forêt de Ouèdo est situé entre $2^{\circ} 16^{\prime}$ et $2^{\circ} 17^{\prime}$ longitude est et entre $6^{\circ} 30^{\prime}$ et $6^{\circ} 32^{\prime}$ latitude nord avec une superficie de 580 ha. Cette forêt est entouré par Kpossidja au sud et nord, Adjagbo à l'est, et par Dassèkomè à l'ouest. Quant à la forêt de Pahou, elle est comprise entre les latitudes $6^{\circ} 22^{\prime}$ ' et $6^{\circ} 27^{\prime}$ nord et les longitudes $2^{\circ} 8^{\prime}$ et $2^{\circ} 14^{\prime}$ Est. Elle est localisée dans l'arrondissement de Pahou et entourée par les villages administratifs d'Ahouicodji, Houndjava au sud, Ahozon au nord-est, Dékouènou au nord et Tovè 2 au nord est avec une superficie de 765 ha (MEPN, 2010). Situées dans la zone sud du Bénin, les forêts classées de Pahou et de Ouèdo appartiennent à une région soumise à un climat de type guinéen ou subéquatorial. Ce climat est caractérisé par deux saisons pluvieuses alternant avec deux saisons sèches: une grande saison pluvieuse de mars à juillet; une petite saison sèche correspondant au mois d'août; une petite saison pluvieuse de septembre à octobre ; une grande saison sèche de novembre à février. La pluviométrie moyenne annuelle de la région calculée sur la période de 1985 à 2016 est de $1147 \mathrm{~mm}$ (METEO-Bénin, 2017).

Le pic des précipitations correspond habituellement au mois de juin. Deux types de sols sont rencontrés à Pahou : les terres de barre et les sables littoraux. Quant à la forêt classée de Ouèdo, elle repose entièrement sur des sols ferralitiques faiblement désaturés appauvris, sur sédiment meuble argilo-sableux du continental terminal (Volkoff et Willaime, 1976). La Figure 1 présente la situation géographique de ces deux forêts dans les communes de Ouidah et d'Abomey-Calavi.

\section{Méthodes de collecte de données}

L'étude a été réalisée dans la zone guinéenne, du Bénin en novembre 2017. La méthode d'estimation est basée sur l'approche développée par Valentini (2007), et basée sur la méthode indirecte utilisant les équations allométriques. Cette recherche s'est déroulée suivant des étapes. 


\section{Collecte des données climatiques}

Pour une analyse de corrélation entre les éléments du climat et les stocks de carbone, les données climatologiques (température $\left({ }^{\circ} \mathrm{C}\right)$ et pluviométrie $(\mathrm{mm})$ ) ont été collectées au niveau de la station météorologique de recherche d'Agonkanmè proche des deux forêts. Les données climatiques ont été collectées sur la base des âges respectifs des plantations (1987-2017).

\section{Mesures biophysiques des biomasses}

Le protocole de mesure est basé sur la réalisation des mesures de la biomasse dans des parcelles de $400 \mathrm{~m}^{2}$. Les parcelles sont de formes rectangulaires car jugées plus hétérogènes et plus représentatives du peuplement. La parcelle permet ainsi d'avoir un aperçu représentatif des plantations sous forme de transect.

\section{Mesure dendrométrique}

Diamètre à hauteur de poitrine $(D B H)$

Il est déterminé à partir de la circonférence mesurée à l'aide d'un mètre ruban, à 1,30 m au-dessus du sol. Ce DBH est calculé par la formule:

$$
\mathrm{DBH}=\frac{\mathrm{C}}{\Pi}
$$

Avec $\mathrm{C}$ : circonférence de l'arbre à $1,30 \mathrm{~m}$ audessus du sol et $\prod=3,14$

\section{Hauteur de l'arbre}

Pour déterminer la hauteur de l'arbre $\mathrm{H}$, deux visées ont été réalisées. Une première au sommet de l'arbre (V1) et un second au pied de l'arbre (V2). Ces visées sont en pourcentage de la distance (L) séparant l'opérateur de l'arbre. La hauteur totale de l'arbre mesurée est obtenue par la relation suivante (Rondeux, 1999):

\section{Densité apparente}

$$
\mathrm{H}=\frac{(\mathrm{V} 2-\mathrm{V} 1) * \mathrm{~L}}{\mathbf{1 0 0}}
$$

La densité apparente sur les différents sites de recherche a été déterminée par la méthode de cylindre. Elle consiste à prélever à l'aide d'un cylindre métallique creux un échantillon de terre. La terre extraite avec le cylindre est arasée aux extrémités. Le volume de terre prélevé est égal à celui du cylindre. La terre est ensuite enlevée et séchée à $105^{\circ} \mathrm{C}$, au Laboratoire des Sciences du Sol, Eau et Environnement (LSSEE, ex. CENAP) de l'Institut National des Recherches Agricoles du Bénin (INRAB), puis pesée.

Densité apparente= Poids du sol séché-poids vide du cylindre

\section{Méthode d'estimation du carbone séquestré}

Dans la présente recherche, la méthode d'estimation du carbone est basée sur les recommandations faites par l'IPCC en 2003 dans le «Good Practice Guidance for Land Use, Land-use Change and Forestry» (LULUCF) ainsi que sur l'ouvrage réalisé par MacDiken en 1997, méthode décrite dans Valentini (2007). Elle a consisté à évaluer la biomasse présente dans plusieurs composantes (aériennes et souterraines), et la matière organique du sol.

Estimation du carbone du sol

La méthode utilisée pour déterminer le carbone du sol consiste à déterminer le carbone organique total à différentes profondeurs ou globalement pour un ou plusieurs horizons, et de transformer les données, en tenant compte de la densité apparente du sol. Les statistiques sont calculées sur différents échantillons afin de déterminer les réserves de carbone. Le résultat est exprimé en total de $\mathrm{kg} \mathrm{C} / \mathrm{m}^{2}, \mathrm{t} \mathrm{C} / \mathrm{ha}$ dans des zones et à des profondeurs spécifiées (FAO, 2002).

\section{Equation}

$$
\begin{aligned}
& \operatorname{COS}=\sum_{\text {horizon }=1}^{\text {horizon }} \text { COShorizon }= \\
& \sum_{\text {horizon }=1}^{\text {horizon }=n}([\operatorname{COS}] * D A * P *(1-\text { frag }) *
\end{aligned}
$$

10)horizon

Avec : $\operatorname{COS}=$ teneur en carbone organique des sols représentative du type de formation végétale et du sol étudié (exprimée en tonne C.ha ${ }^{-1}$ ) ; COS horizon = teneur en carbone organique pour un horizon constitutif du sol (tonne C.ha ${ }^{-1}$ ) ; [COS $]=$ concentration $\mathrm{du}$ carbone organique dans une masse de sol 
donnée, obtenue par analyse au laboratoire (g $\mathrm{C} \mathrm{kg} / \mathrm{sol}$ ) ; DA = Densité Apparente (tonne de $\mathrm{sol} / \mathrm{m} 3) ; \mathrm{P}=$ profondeur de l'horizon ou épaisseur de la couche du sol (m) ; frag = pourcentage de volume de fragments grossiers/100.

Estimation du stock de carbone dans la biomasse aérienne et racinaire

Mesure sur le terrain de la biomasse aérienne vivante des Acacia auriculiformis

Pour estimer la biomasse des grands arbres, il a été recommandé d'utiliser l'équation de la FAO (1997) pour les zones arides $(<1500 \mathrm{~mm}$ par an). Pour estimer la biomasse aérienne et racinaire présente dans chaque arbre, les équations suivantes ont été utilisées.

Equation 2: $\mathrm{BA}=\exp ^{(-1,996+2,32 \ln D)} \quad(\mathrm{D}$ en $\mathrm{cm}$ )

Equation 3: $\mathrm{BR}=\exp ^{(-1,0587+0,8836 * \ln (B A))}$

La détermination de la quantité de carbone présente dans les différentes composantes de l'arbre et dans chaque plantation a été faite en se basant sur les équations allométriques de la FAO (1997) et adapté (Tableau 1) selon les modèles du stock de carbone pour les différentes composantes des arbres par Boulmane et al. (2013).

\section{Analyse chimique}

Sol

Sous chaque arbre identifié et sous clairière, les échantillons de sol ont été prélevés à $0-20 \mathrm{~cm}, 20-40 \mathrm{~cm}$ et $40-60 \mathrm{~cm}$ de profondeur suivant trois différents points afin de constituer des échantillons composites. Au total, 72 échantillons de sols ont été prélevés (soit 3 arbres $\mathrm{x} 3$ profondeurs et 3 prélèvements d'échantillon pour la densité apparente $\mathrm{x} 8$ zones de recherche).

Au laboratoire, les échantillons de sol ont été d'abord séchés à la température ambiante puis tamisés à $2 \mathrm{~mm}$ en vue de séparer les éléments fins et ceux grossiers. Ces échantillons ont servi à déterminer le fragment, et pour les analyses chimiques. Ces analyses ont consisté en la détermination du carbone organique par la méthode de Walkley \& Black qui consiste à oxyder la matière organique du sol avec le dichromate de potassium $(\mathrm{K} 2 \mathrm{Cr} 2 \mathrm{O} 71 \mathrm{~N})$ en milieu acide dans le rapport sol/K2Cr2O de 0,25/10. La teneur en carbone est déterminée par titrage avec une solution de sulfate de fer $0,5 \mathrm{~N}$ après ajout d'indicateur de diphénylamine.

Végétation herbacée et litière

Sur chaque site, trois quadrats de 1 $\mathrm{m}^{2}$ ont été délimités dans chaque parcelle principale. Toute la végétation présente dans chaque quadrat a été coupée puis la litière se trouvant dans chaque quadrat a été collectée et pesée sur place. Ensuite des échantillons de végétation et de litière sont prélevés, et ramenés au laboratoire pour les analyses chimiques. Les échantillons de litière et de végétaux herbacés séchés à l'étuve à $65{ }^{\circ} \mathrm{C}$ ont été broyés pour la détermination du carbone organique par la méthode de calcination. Le carbone a été déterminé par incinération au four à $525^{\circ} \mathrm{C}$, après le passage à l'étuve à $105^{\circ} \mathrm{C}$ pendant 48 heures. Ceci permet de déterminer le taux de matière organique qui est ensuite convertie en carbone organique par la formule suivante : \% Corg = $\% \mathrm{MO} / 1,724$ (avec Corg = Carbone organique et $\mathrm{MO}=$ matière organique)

\section{Analyse statistique}

Le tableur Excel a été utilisé pour la saisie et le traitement des données. Le logiciel Statistical Analysis System version 9.2 (SAS v. 9.2) a été ensuite utilisé pour les analyses statistiques. Ces analyses ont essentiellement consisté en des analyses de variance (à un et deux facteurs). Les valeurs moyennes ont été ensuite comparées entre elles à l'aide du test de la plus petite différence significative au seuil de 5\% (niveau de probabilité). 


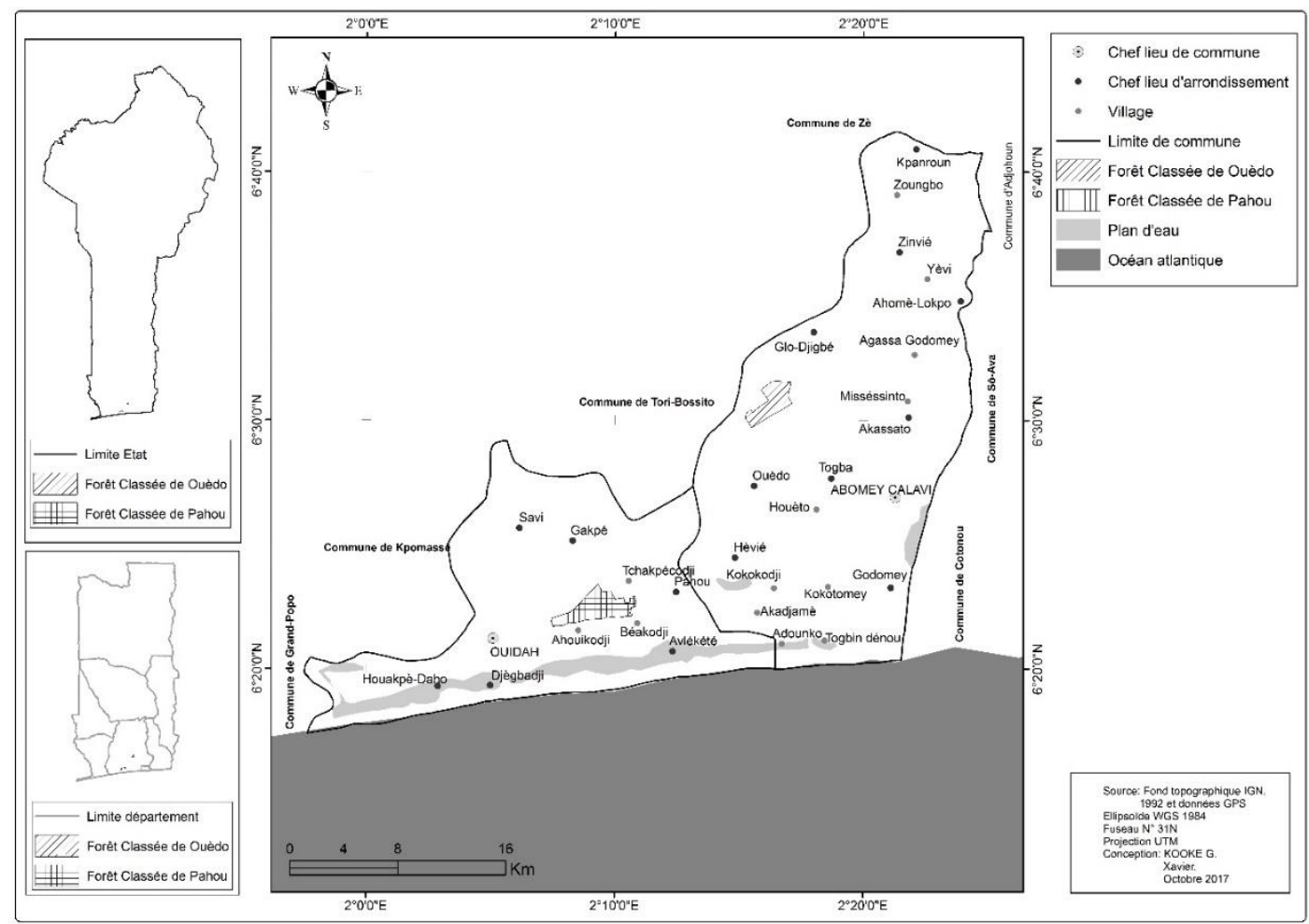

Figure 1 : Situation géographique des forêts classées de Pahou et de Ouèdo au sud du Bénin

Tableau 1: Modèles de stocks de carbone pour les différentes composantes des arbres.

\begin{tabular}{ll}
\hline Composante de l'arbre & Estimation du stock de carbone exprimée en kg/arbre \\
\hline Tronc & $\mathrm{SCTr}=191 \times\left(\mathrm{D}^{2} \mathrm{H}\right)$ \\
Branche & $\mathrm{SCBr}=46 \times\left(\mathrm{D}^{2} \mathrm{H}\right)$ \\
Feuille & $\mathrm{SCF}=5,4 \times\left(\mathrm{D}^{2} \mathrm{H}\right)$
\end{tabular}

Avec $\mathrm{D}$ : diamètre à $1,30 \mathrm{~m}, \mathrm{H}$ : hauteur de l'arbre en $\mathrm{m}$. D et $\mathrm{H}$ en $\mathrm{m}$. SCTr: stock de carbone dans le tronc, SCBr: stock de carbone dans les branches, SCF: stock de carbone dans les feuilles.

\section{RESULTATS}

\section{Stock de carbone organique dans les différents compartiments de Acacia auriculiformis}

Les différents compartiments de Acacia auriculiformis étudiés tiennent compte de la biomasse aérienne (tronc, branches et feuilles) et biomasse racinaire (racines). Le stock de carbone varie significativement $(\mathrm{P}<0,05)$ selon les différents compartiments des Acacia auriculiformis. En fonction de ces compartiments, le stock de carbone est plus élevé dans le tronc, $78,9 \%$ de la biomasse aérienne, les branches : 19\% de la biomasse aérienne et les feuilles: $2,1 \%$ de la biomasse aérienne. Les résultats des stocks de carbone organique dans les différents compartiments des Acacia auriculiformis sont consignés dans le Tableau 2. De l'analyse de ce tableau, il est noté qu'au niveau des plantations, il y a une différence significative $(P<0,05)$ entre les âges des plantations et les types de sol pour ce qui concerne, les stocks de carbone contenu dans le tronc, les branches et les feuilles. 
Globalement, entre la biomasse aérienne et racinaire, il n'est noté aucune différence significative $(P>0,05)$ en ce qui concerne les stocks de carbone des différents âges et des types de sol. Les valeurs les plus élevées du stock de carbone sont observées de façon décroissante en partant des plantations d'âge avancé vers les plantations d'âge plus bas. Il est donc à noter que l'âge influence la capacité de séquestration du carbone sur même type de sol. Cependant, il est noté une différence significative $(\mathrm{P}<0,05)$ du stock de carbone séquestré dans les plantations de même âge et sur des sols différents. En effet, Les résultats obtenus dans les plantations de 2,5 ans sur sol ferralitique et hydromorphe à Pahou sont différents. Les sols ferralitiques ont une capacité de séquestration de carbone plus élevé que les sols hydromorphes sous plantation de Acacia auriculiformis. En considérant uniquement l'âge, que ce soit au niveau des troncs, des branches, des feuilles et racinaires, les valeurs les plus élevée, du stock de carbone ont été observées dans les plantations les plus âgées comparativement aux plantations de petit âge qui présentent les valeurs de stock de carbone les plus faibles dans chaque plantation.

D'une manière générale, les plantations de Acacia auriculiformis âgé de 6 ans sur sol ferralitique à Ouèdo ont plus stocké de carbone dans les différents compartiments des arbres alors que celles de la même espèce (Acacia auriculiformis) âgé de 2,5 ans sur sol ferrugineux tropicaux lessivé à Pahou, de Acacia auriculiformis âgé de 2,5 ans sur sol hydromorphe et de Acacia auriculiformis âgé de 2 ans sur sol ferralitique à Ouèdo ont le moins stocké selon le test de la plus petite différence significative. Par contre, selon le même test, la forêt naturelle de Pahou a plus stocké de carbone dans tous les compartiments que tous les différents âges de plantation à Pahou sur même type de sol.

\section{Teneur et stock de carbone organique du sol dans les plantations}

L'évaluation du stock de carbone dans le sol ne tient pas compte de la matière organique au-dessus de la surface du sol. La teneur en carbone du sol dans les plantations de Acacia auriculiformis a diminué en fonction de la profondeur de prélèvement. L'horizon 0-20 cm représente en moyenne plus de $51 \%$ du stock total de carbone du sol quelle que soit la zone d'étude (Figure 2). Il ressort de l'analyse de cette Figure 2 que quels que soient la zone, l'âge et le type de sol, les valeurs les plus élevées des teneurs en carbone et stock de carbone organique du sol ont été observées dans l'horizon 0-20 cm.

L'horizon 40 à $60 \mathrm{~cm}$ présente les valeurs des teneurs en carbone et stock de carbone organique du sol les plus faibles. En considérant les zones et les âges, il ressort de l'analyse de la Figure 2 que dans la plantation de 2,5 ans sur sol hydromorphe à Pahou, aucune différence significative $(\mathrm{P}>0,05)$ n'est observée entre les différents horizons en ce qui concerne les teneurs en carbone et le stock de carbone organique du sol.

Le même constat est observé au niveau de la plantation de 6 ans sur sol ferralitique à Ouèdo pour ce qui concerne les teneurs en stock de carbone organique du sol. Cependant, la plantation de 2,5 ans sur sol hydromorphe à Pahou a enregistré la teneur en carbone et le stock de carbone organique du sol, la plus élevée. Dans la plantation de 2,5 ans sur sol hydromorphe à Pahou les valeurs des teneurs en carbone et stock de carbone organique du sol sont les plus élevées comparativement à la plantation de 2 ans sur sol ferralitique à Ouèdo qui présente les valeurs les plus faibles. En conséquence, le sol hydromorphe a plus de capacité de séquestration de carbone que le sol ferralitique. 
Au niveau des deux forêts, aucune différence significative $((P>0,05)) \quad$ n'est observée entre les différents âges pour les teneurs en carbone et pour les stocks de carbone organique du sol, par contre, en considérant les types de sol et les deux forêts, la plantation sur sol hydromorphe présente la plus forte valeur comparativement aux autres plantations de même espèce (Acacia auriculiformis) qui présentent les valeurs les plus faibles (Tableau 3). D'une manière générale, les plantations de Ouèdo sur même type de sol et même âge et celles de Pahou présentent les valeurs des teneurs en carbone les plus élevées comparativement à celles de Pahou qui présentent les plus faibles valeurs. Pour ce qui concerne le stock de carbone organique du sol, c'est aussi les plantations de Ouèdo sur même type de sol et même âge qui présentent les valeurs les plus élevées comparativement à celle de Pahou qui présentent les plus faibles valeurs.

\section{Stock de carbone organique dans la litière et la végétation herbacée dans les plantations}

Le Tableau 4 présente les valeurs moyennes du stock de carbone dans la biomasse herbacée et dans la litière au niveau des plantations de Ouèdo et de Pahou. Il ressort de l'analyse de ce tableau qu'il n'y a pas de différence significative $(\mathrm{P}>0,05)$ entre les forêts en ce qui concerne le stock de carbone dans la biomasse herbacée. Le test de la plus petite différence significative montre que les plantations de 12 ans sur sol ferrugineux lessivés tropicaux ont plus significativement $(\mathrm{p}<0,05)$ stocké le carbone dans la litière pendant que les autres plantations ont stocké moins.

\section{Stock de carbone dans le sol et stock total dans les plantations}

Le Tableau 5 présente les valeurs moyennes des stocks de carbone total dans le sol et dans les plantations selon l'âge et le type de sol. De l'analyse du tableau, il est noté qu'au niveau des plantations de Pahou hormis celle de 2,5 ans d'âge sur sol hydromorphe, aucune différence significative $(\mathrm{P}>0,05)$ n'est observée entre les différents âges et même types de sol en ce qui concerne le stock de carbone organique dans le sol. Par contre, le stock de carbone total dans ces plantations varie significativement. Le même constat est fait au niveau des plantations de 4 et 6 ans d'âge sur sol ferralitique. Globalement, les plantations de 2,5 ans sur sol hydromorphe à Pahou et celle de 6 ans sur sol ferralitique à Ouèdo présentent les valeurs les plus élevées de stock de carbone total dans le sol tandis que les plantations de 4 ans sur sol ferralitique à Pahou présentent les valeurs les plus faibles du stock total de carbone dans les plantations.

\section{Corrélation entre facteurs climatiques et stock de carbone dans les plantations de Acacia auriculiformis}

Les moyennes des températures et des hauteurs pluviométriques obtenues au cours des années correspondantes aux âges des plantations de Acacia auriculiformis ( 2 ; $3 ; 4 ; 6 ; 12$ et 30 ans) ont été mises en relation avec les données de stock de carbone organique total obtenu au niveau des plantations de Acacia auriculiformis des différentes zones parcourues. Cette relation montre qu'il existe une corrélation négative et très hautement significative $(\mathrm{r}=-0,931 ; \mathrm{P}<$ 0,001 ) entre le stock de carbone et la température. Aucune corrélation significative $(r=-0,186 ; \mathrm{P}>0,05)$ n'est obtenue cependant entre le stock de carbone organique et la moyenne des hauteurs pluviométriques. Il en résulte que les fortes températures observées dans le milieu de recherche sont donc le résultat de la faible séquestration de carbone dans le milieu et vice-versa. 
G. X. KOOKE et al. / Int. J. Biol. Chem. Sci. 13(1): 277-293, 2019

Tableau 2: Stocks de carbone contenu dans la biomasse aérienne et racinaire (valeurs moyennes \pm erreur standard).

\begin{tabular}{|c|c|c|c|c|c|c|c|}
\hline \multirow[t]{2}{*}{ Espèces } & \multirow[t]{2}{*}{ Type de sol } & \multirow{2}{*}{$\begin{array}{l}\text { Age } \\
\text { (ans) }\end{array}$} & \multicolumn{5}{|c|}{ Stock de carbone dans } \\
\hline & & & Tronc (t C/ha) & Branches (t C/ha) & Feuilles (t C/ha) & Biomasse aérienne (t C/ha) & Biomasse racinaire (t C/ha) \\
\hline \multirow{7}{*}{$\begin{array}{l}\text { Acacia } \\
\text { auriculiformis }\end{array}$} & Ferrugineux tropicaux & 12 & $20,77 \pm 02,86 b$ & $05,00 \pm 0,67 \mathrm{~b}$ & $0,59 \pm 0,08 \mathrm{~b}$ & $31,99 \pm 4,00 \mathrm{ab}$ & $13,11 \pm 01,21 \mathrm{a}$ \\
\hline & lessivé & 4 & $20,46 \pm 5,24 b$ & $4,93 \pm 1,26 \mathrm{~b}$ & $0,58 \pm 0,15 b$ & $28,38 \pm 6,52 \mathrm{ab}$ & $10,00 \pm 02,03 \mathrm{a}$ \\
\hline & (Pahou) & 2,5 & $18,20 \pm 05,47 \mathrm{~b}$ & $04,38 \pm 1,32 b$ & $0,51 \pm 0,15 \mathrm{~b}$ & $27,14 \pm 7,46 b$ & $09,04 \pm 02,90 \mathrm{a}$ \\
\hline & & 6 & $42,51 \pm 06,75 \mathrm{a}$ & $10,24 \pm 1,62 \mathrm{a}$ & $1,29 \pm 0,19 \mathrm{a}$ & $50,11 \pm 7,14 \mathrm{a}$ & $26,09 \pm 13,55 \mathrm{a}$ \\
\hline & Ferralitique & 4 & $20,31 \pm 13,41 \mathrm{ab}$ & $06,34 \pm 3,23 \mathrm{ab}$ & $0,75 \pm 0,38 \mathrm{ab}$ & $31,52 \pm 15,28 \mathrm{ab}$ & $16,28 \pm 09,62 \mathrm{a}$ \\
\hline & (Ouèdo) & 2 & $11,72 \pm 03,40 \mathrm{~b}$ & $02,82 \pm 0,82 b$ & $0,33 \pm 0,10 \mathrm{~b}$ & $19,59 \pm 5,13 b$ & $10,14 \pm 01,43 \mathrm{a}$ \\
\hline & hydromorphe (Pahou) & 2,5 & $09,58 \pm 03,77 \mathrm{~b}$ & $02,31 \pm 0,91 \mathrm{~b}$ & $0,27 \pm 0,11 \mathrm{~b}$ & $14,48 \pm 5,40 \mathrm{~b}$ & $10,24 \pm 03,93 \mathrm{a}$ \\
\hline $\begin{array}{l}\text { Terminalia } \\
\text { superba }\end{array}$ & $\begin{array}{l}\text { ferrugineux tropicaux } \\
\text { lessivés }\end{array}$ & $\begin{array}{l}\text { Noyau } \\
\text { central }\end{array}$ & $24,91 \pm 03,54 \mathrm{ab}$ & $06,00 \pm 0,85 \mathrm{ab}$ & $0,70 \pm 0,10 a b$ & $33,36 \pm 4,26 a b$ & $12,33 \pm 02,12 \mathrm{a}$ \\
\hline Probabilité & & & 0,04 & 0,04 & 0,04 & 0,1 & 0,51 \\
\hline
\end{tabular}

Les moyennes suivies des mêmes lettres alphabétiques de même caractères et pour les mêmes caractéristiques ne sont pas significativement différentes $(\mathrm{P}>0,05)$ d'après le test de la plus petite différence significative. 


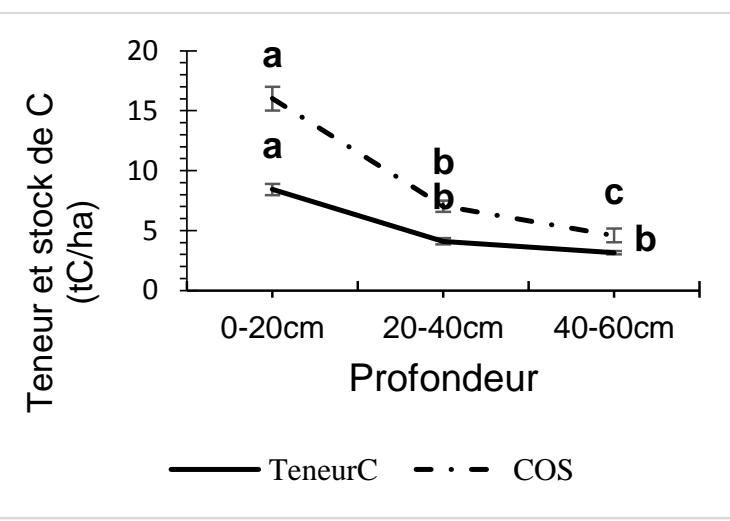

Acacia a. de 12 ans à Pahou

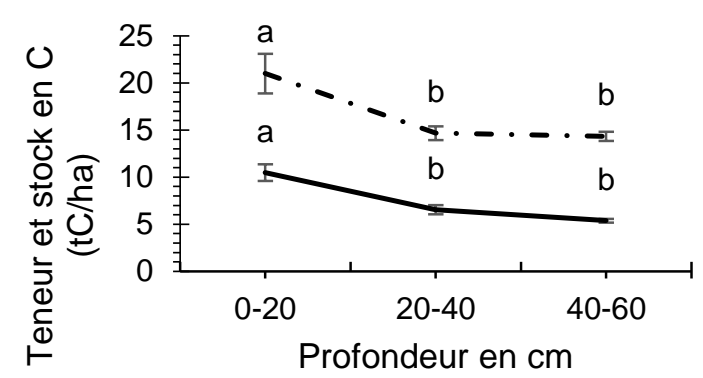

- TeneurC $-\cdot-\cos$

Acacia a. de 4 ans à Ouèdo sur sol ferralitique

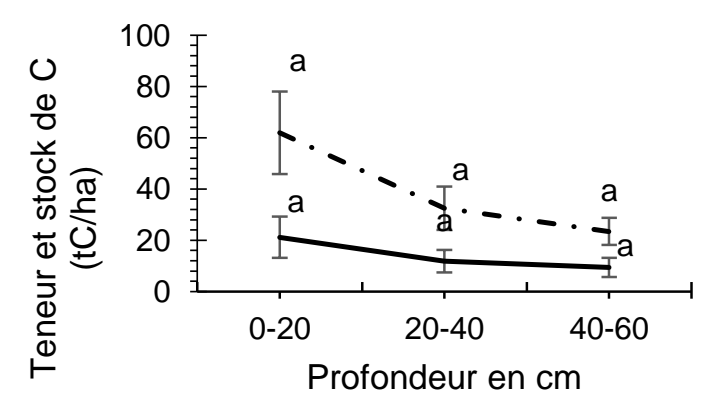

— TeneurC - - c cos

Acacia a. de 2,5 ans à Pahou sur sol hydromorphe

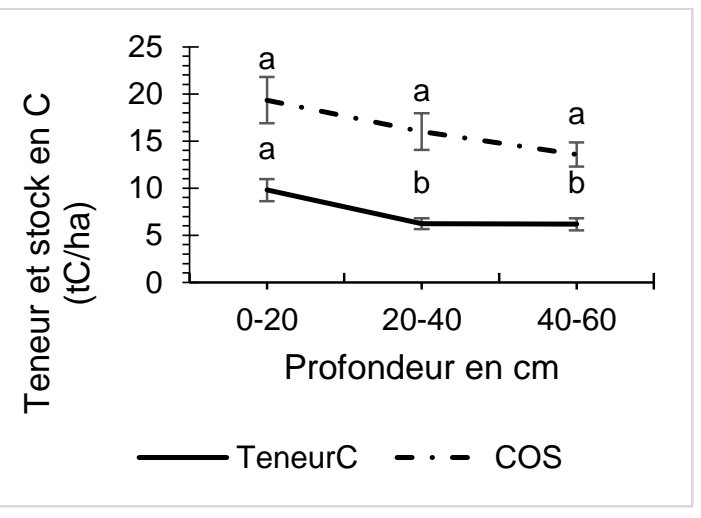

Acacia a. de 6 ans à Pahou sur sol ferralitique

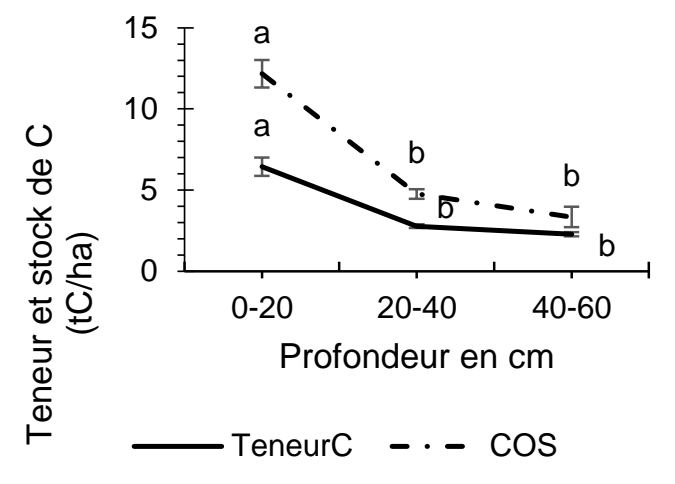

Acacia a. de 4 ans à Pahou

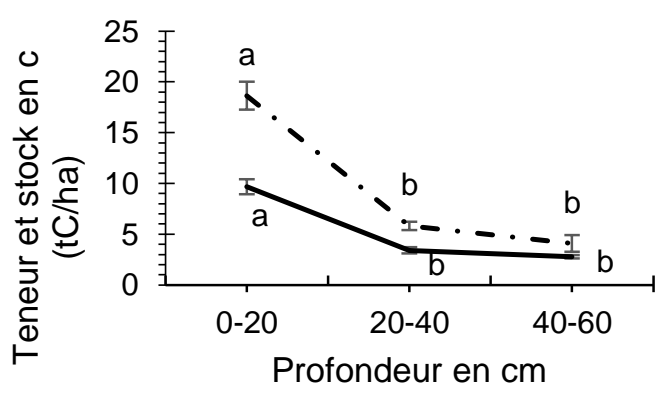

- TeneurC $-\cdots \cos$

Acacia a. de 2,5 ans à Pahou 


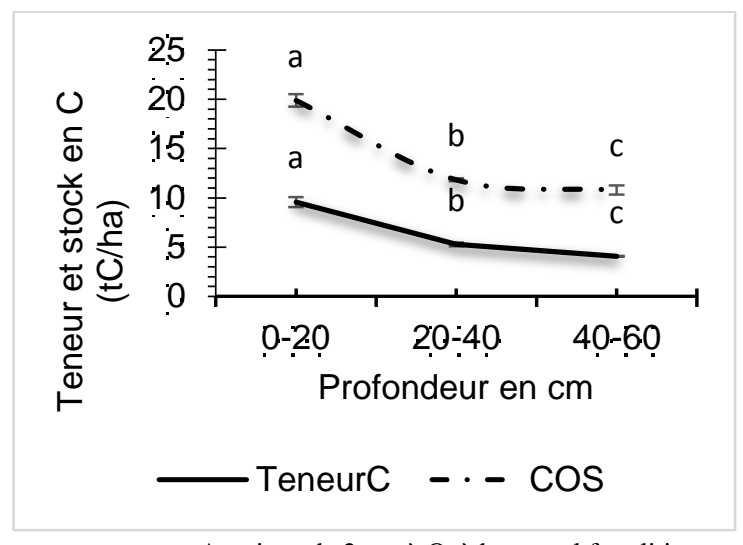

Acacia a. de 2 ans à Ouèdo sur sol ferralitique

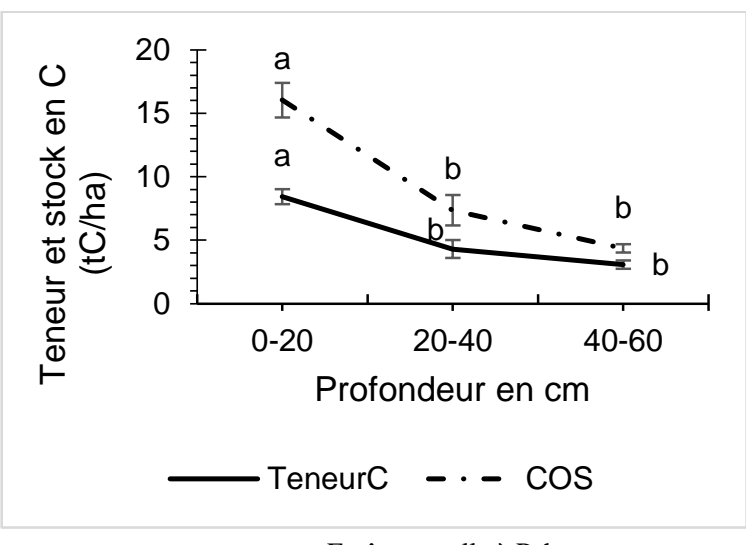

Forêt naturelle à Pahou

Figure 2 : Répartition du stock de carbone dans les plantations de Acacia auriculiformis suivant les différents horizons du sol et en fonction des âges et des types de sol (valeurs moyennes \pm erreur standard).

Tableau 3 : Effet des différents âges des plantations étudiées, du type de sol et de l'âge sur la moyenne des teneurs en carbone et stock de carbone organique du sol.

\begin{tabular}{|c|c|c|c|c|}
\hline Espèces & Type sol & $\begin{array}{l}\text { Age } \\
\text { (ans) }\end{array}$ & $\begin{array}{c}\text { Teneur en } \\
\text { carbone (t } \mathrm{C} / \mathrm{ha})\end{array}$ & $\begin{array}{c}\text { Stock de carbone } \\
\text { organique du sol (tC/ha) }\end{array}$ \\
\hline \multirow{7}{*}{$\begin{array}{l}\text { Acacia } \\
\text { auriculiformis }\end{array}$} & Ferrugineux tropicaux & 12 & $5,23 \pm 0,83 b c$ & $9,21 \pm 0,77 \mathrm{dc}$ \\
\hline & lessivé & 4 & $3,82 \pm 0,68 \mathrm{c}$ & $6,75 \pm 1,41 \mathrm{~d}$ \\
\hline & (Pahou) & 2,5 & $5,28 \pm 1,13 \mathrm{bc}$ & $9,41 \pm 2,03 \mathrm{dc}$ \\
\hline & Ferralitique & 6 & $7,40 \pm 0,73 b$ & $16,30 \pm 1,29 b$ \\
\hline & (Ouèdo) & 4 & $7,45 \pm 0,83 b$ & $16,66 \pm 1,27 b$ \\
\hline & & 2 & $6,30 \pm 0,85 b c$ & $14,15 \pm 1,45 b c$ \\
\hline & Hydromorphe (Pahou) & 2,5 & $14,14 \pm 3,37 \mathrm{a}$ & $25,14 \pm 6,79 a$ \\
\hline $\begin{array}{l}\text { Terminalia } \\
\text { superba }\end{array}$ & $\begin{array}{l}\text { Ferrugineux tropicaux } \\
\text { lessivé (Pahou) }\end{array}$ & $\begin{array}{l}\text { Noyau } \\
\text { central }\end{array}$ & $5,26 \pm 0,86 b c$ & $9,25 \pm 1,85 \mathrm{dc}$ \\
\hline Probabilité & & & 0,0001 & 0,0001 \\
\hline
\end{tabular}

Les Moyennes suivies des mêmes lettres alphabétiques de même caractères et pour les mêmes caractéristiques ne sont pas significativement différentes $(\mathrm{P}>0,05)$ d'après le test de la plus petite différence significative.

Tableau 4 : Valeur moyenne (Moyennes \pm erreur standard) du stock de carbone dans la biomasse herbacée et litière dans les différentes plantations de Acacia auriculiformis.

\begin{tabular}{lllcc}
\hline Espèces & Type sol & Age (ans) & $\begin{array}{c}\text { Biomasse } \\
\text { Herbacée }(\mathbf{t C} / \mathbf{h a})\end{array}$ & Litière (tC/ha) \\
\hline Acacia & Ferrugineux tropicaux & 12 & $3,08 \pm 0,04 \mathrm{a}$ & $2,98 \pm 0,10 \mathrm{a}$ \\
auriculiformis & lessivé & 4 & $3,13 \pm 0,02 \mathrm{a}$ & $2,60 \pm 0,08 \mathrm{ab}$ \\
& (Pahou) & 2,5 & $3,16 \pm 0,03 \mathrm{a}$ & $2,07 \pm 0,31 \mathrm{c}$ \\
\cline { 2 - 5 } & Ferralitique & 6 & $3,07 \pm 0,03 \mathrm{a}$ & $2,65 \pm 0,16 \mathrm{ab}$ \\
& (Ouèdo) & 4 & $3,13 \pm 0,04 \mathrm{a}$ & $2,87 \pm 0,11 \mathrm{ab}$ \\
& 2 & $3,16 \pm 0,01 \mathrm{a}$ & $2,42 \pm 0,02 \mathrm{bc}$ \\
\hline
\end{tabular}




\begin{tabular}{lllll} 
& Hydromorphe (Pahou) & 2,5 & $3,16 \pm 0,01 \mathrm{a}$ & $2,93 \pm 0,05 \mathrm{ab}$ \\
\hline $\begin{array}{l}\text { Terminalia } \\
\text { superba }\end{array}$ & $\begin{array}{l}\text { Ferrugineux tropicaux } \\
\text { lessivé (Pahou) }\end{array}$ & Noyau central & $3,13 \pm 0,03 \mathrm{a}$ & $2,07 \pm 0,25 \mathrm{c}$ \\
\hline Probabilité & & & & \\
\hline
\end{tabular}

Les moyennes suivies des mêmes lettres alphabétiques de même caractères et pour les mêmes caractéristiques ne sont pas significativement différentes $(\mathrm{P}>0,05)$ d'après le test de la plus petite différence significative.

Tableau 5 : Effet des différents âges des plantations étudiées et du type de sol sur le stock de carbone total et du carbone organique total du sol (Moyennes \pm erreur standard).

\begin{tabular}{|c|c|c|c|c|}
\hline Espèces & Type_sol & Age (ans) & $\begin{array}{c}\text { stock de carbone } \\
\text { organique du sol (t } \\
\text { C/ha) }\end{array}$ & $\begin{array}{c}\text { Stock de } \\
\text { Carbone Total } \\
\text { (t C/ha) }\end{array}$ \\
\hline \multirow{7}{*}{$\begin{array}{l}\text { Acacia } \\
\text { auriculiformis }\end{array}$} & Ferrugineux tropicaux & 12 & $27,63 \pm 1,87 \mathrm{~b}$ & $78,79 \pm 5,30 \mathrm{ab}$ \\
\hline & lessivés & 4 & $20,25 \pm 1,14 b$ & $64,36 \pm 8,58 b$ \\
\hline & (Pahou) & 2,5 & $28,24 \pm 1,95 b$ & $69,65 \pm 9,99 \mathrm{~b}$ \\
\hline & Ferralitique & 6 & $48,91 \pm 5,18 \mathrm{ab}$ & $130,83 \pm 16,39 \mathrm{a}$ \\
\hline & (Ouèdo) & 4 & $49,97 \pm 3,03 \mathrm{ab}$ & $\begin{array}{c}103,77 \pm 26,45 \\
a b\end{array}$ \\
\hline & & 2 & $42,46 \pm 1,09 b$ & $77,77 \pm 5,47 \mathrm{ab}$ \\
\hline & Hydromorphe (Pahou) & 2,5 & $75,42 \pm 29,73 \mathrm{a}$ & $\begin{array}{c}106,23 \pm 38,39 \\
\mathrm{ab}\end{array}$ \\
\hline $\begin{array}{l}\text { Terminalia } \\
\text { superba }\end{array}$ & $\begin{array}{l}\text { Ferrugineux tropicaux } \\
\text { lessivés (Pahou) }\end{array}$ & $\begin{array}{l}\text { Noyau } \\
\text { central }\end{array}$ & $27,75 \pm 1,57 b$ & $78,66 \pm 4,55 \mathrm{ab}$ \\
\hline Probabilité & & & 0,0691 & 0,3236 \\
\hline
\end{tabular}

\section{DISCUSSION}

Séquestration de carbone dans les plantations de Acacia auriculiformis de Ouèdo et de Pahou

La présente recherche a révélé que le stock de carbone dans les plantations de Acacia auriculiformis (Pahou et Ouèdo) varie en fonction de l'âge et du type de sol. En effet, à Ouèdo, sur un même type de sol (ferralitique), les Acacia auriculiformis les plus âgés ont stocké plus de carbone pour la plupart. Sur le même type de sol (ferralitique ou hydromorphe), le stock de carbone évolue $\mathrm{du}$ plus grand au plus petit âge. Selon Thompson et al. (2003), plus l'arbre grandit, plus il séquestre du carbone. Les arbres plantés sur des sols hydromorphes ont plus stocké de carbone que ceux plantés sur des sols ferralitiques.

Le stock de carbone est significativement plus élevé dans la biomasse aérienne que dans la biomasse racinaire. Ce résultat corrobore celui de Boulmane et al. (2013); de Bello et al. (2017) et Diatta et al. (2016) qui ont obtenu des résultats similaires au Maroc dans la chênaie verte, au Bénin sur plantation d'anacardier et au Sénégal dans le Sud du Bassin Arachidier. Montagnini et Nair (2004), ont montré que les efforts de captation de carbone en milieu tropical sont concentrés sur la biomasse aérienne et non sur les sols. Selon ces auteurs, en 25 ans, les stocks de carbone dans la végétation peuvent augmenter de $50 \mathrm{tC} / \mathrm{ha}$, comparativement à seulement 5 à $15 \mathrm{tC} / \mathrm{ha}$ dans les sols (le taux de 
décomposition de la matière organique du sol étant très rapide sous ces climats).

Selon les différentes plantations, le stock de carbone dans la biomasse aérienne varie entre $14,48 \pm 5,40 \mathrm{tC} /$ ha et $50,11 \pm 7,14$ tC/ha. Ces résultats sont similaires à ceux obtenus par Albrecht et Kandji (2003) et Bello et al. (2017) qui l'estiment entre 7 et $25 \mathrm{tC} / \mathrm{ha}$ dans un système agroforestier. Ces valeurs sont dans l'intervalle donné par Palm et al. (2000), entre 40 et $60 \mathrm{t} \mathrm{C} / \mathrm{ha}$ pour les systèmes agroforestiers en milieu tropical. Ce stock de carbone obtenu est supérieur à celui obtenu au niveau des parcs à karité et néré de Bemberékè 24,42 $\pm 6,98 \mathrm{t} \mathrm{C}$ /ha (Medlyn et al., 2013). Le stock de carbone dans le sol obtenu varie entre $09,04 \pm 02,90$ et 26,09 \pm $13,55 \mathrm{tC} / \mathrm{h}$. Seules les valeurs obtenues dans les plantations de 4 ans, de 2,5 ans, dans la forêt naturelle et de 2 ans à Ouèdo se situent dans l'intervalle des résultats ( 3 à $13 \mathrm{tC} / \mathrm{ha}$ ) obtenus par Valentini (2007). Mouwembe et al. (2017) ont montré que, les stocks de carbone des sols dans les savanes des réserves de Lésio-Louna et de Léfini, plateaux Tékés, République du Congo varient selon les horizons. De ces résultats, Il en ressort que la somme des stocks de carbone organique du sol pour l'horizon $0-20 \mathrm{~cm}$ dans cet écosystème est de $12,80 \mathrm{~kg} . \mathrm{m}-2$ pour LésioLouna et 14,03 kg.m-2 pour Léfini. Ainsi, les stocks de carbone obtenus à travers la présente recherche variant entre $13,11 \pm 01,21$ et 26,09 $\pm 13,55 \mathrm{tC} / \mathrm{ha}$ corroborent ceux de Mouwembe et al. (2017). Ces valeurs varient en fonction du type de sol et des conditions climatiques. Par contre, les résultats de cette recherche sont inférieurs à ceux estimés par IPCC (2003) qui est de $31 \mathrm{tC} / \mathrm{ha}$ pour les zones tropicales sèches et ceux obtenus par Palm et al. (2000) dans un système agroforestier basé sur le cacao qui est de 42 tC/ha. Le stock de carbone dans la matière organique morte (litière) obtenu lors de cette recherche varie entre $2,07 \pm 0,25 \mathrm{tC} /$ ha et 2,98 $\pm 0,10 \mathrm{tC} / \mathrm{ha}$. Ce résultat est proche de ceux obtenus par IPCC (2003) qui estime la teneur en carbone de la matière organique morte à 2,8 tC/ha et que celle-ci peut varier entre 2 à 3 tC/ha. Le stock de carbone total obtenu dans les plantations de Acacia auriculiformis varie entre $64,36 \pm 8,58$ et $130,83 \pm 16,39 \mathrm{tC} / \mathrm{ha}$. Selon Albrecht et Kandji (2003), la capacité de stockage du carbone d'un système agroforestier varie entre 12 et $228 \mathrm{t} \mathrm{C} /$ ha avec une valeur moyenne de $95 \mathrm{tC} / \mathrm{ha}$. Les valeurs obtenues lors de cette recherche sont comprises dans cet intervalle. La variation de stock de $\mathrm{C}$ dans les différentes plantations seraient dues donc à la variation de densité de plantation. La quantité de carbone séquestrée par le système agroforestier dépend des espèces mises en place, leur densité, de la structure et de la fonction de ce dernier. Montagnini et Nair (2004) précisent que la quantité de carbone séquestrée est fonction des espèces d'arbres, des régions géographiques (climat, sol), des densités de plantation et de la gestion du système. Les résultats obtenus sont par contre, inferieurs à l'intervalle de 60 et 90 tC/ha pour une agroforêt de 20 ans proposé par Palm et al. (2000). Une comparaison du stock de carbone dans les plantations de Acacia auriculiformis avec celui du système agricole montre que le stock de carbone dans le système agroforestier est très élevé à celui du système agricole qui n'est exprimé que dans le sol. Même le stock de carbone dans le sol de la plantation dépasse la valeur estimée pour le système agricole. Ceci permet donc d'affirmer que le système agroforestier est un puit de carbone par rapport à un système agricole.

\section{Influence du climat sur le stock de carbone dans les plantations de Acacia auriculiformis}

Les résultats de cette recherche montrent que la température a une corrélation hautement négative et significative avec le stock de carbone dans les plantations de Acacia auriculiformis. Il en résulte qu'une quantité importante de carbone séquestré dans les plantations d'étude induit une réduction de 
l'émission du $\mathrm{CO}_{2}$ atmosphérique atténuant ainsi l'effet de la température. Ces résultats sont en accord avec ceux obtenus par Bello et al. (2017) avec les plantations d'anacardier. Le stock de carbone élevé obtenu à Ouèdo peut s'expliquer par une fixation et un stockage du carbone par les plantations de Acacia auriculiformis. Selon Arrouays et al. (2002), l'augmentation de la température conduit à un déstockage du carbone des sols. Selon Fissore et al. (2008), la température élevée induit une forte activité des microorganismes, entrainant une oxydation de la matière organique qui affecte le $\mathrm{CO}_{2}$ de l'atmosphère. La faible température ralentit le taux de minéralisation de la matière organique constituant le stock de carbone important du sol (FAO, 2012). De façon générale, la stimulation de la productivité des plantes avec des températures plus élevées augmentera l'apport de carbone dans la litière et dans les sols induisant un impact sur l'environnement par un plus fort relargage de $\mathrm{CO}_{2}$ dans l'atmosphère (Reichstein, 2007).

L'accroissement des concentrations des gaz à effet de serre (GES) dans l'atmosphère est maintenant reconnu pour être la principale cause des changements climatiques qui, selon plusieurs études, se traduiront par une augmentation de la température terrestre et de la fréquence d'événements météorologiques extrêmes, ainsi que par une élévation du niveau des océans (Boer et al., 2000).

\section{Conclusion}

La présente recherche révèle que de 1987 à 2017, les paramètres climatiques dans les zones de recherche sont marqués par une fluctuation annuelle. Ainsi, la pluviométrie présente une tendance régressive. Dans toutes les forêts d'étude la température montre une tendance ascendante. Selon les différents compartiments à considérer dans la plantation, le sol et la biomasse aérienne stockent des quantités élevées de carbone.
Néanmoins, la biomasse herbacée avec un faible stock joue un rôle considérable dans le potentiel de séquestration du sol en limitant les feux de brousse qui détruisent la structure du sol. Au vu des résultats obtenus, les plantations de Acacia auriculiformis pourraient profiter le pays du marché mondial du carbone qui augmenterait leurs revenus financiers. Aussi, faut-il mettre en place des politiques pour développer des systèmes agraires durables via l'implantation de projets de séquestration du carbone liés aux changements climatiques sur Acacia auriculiformis.

\section{CONFLIT D'INTERETS}

Les auteurs déclarent qu'il n'y a aucun conflit d'intérêts. Le payement des frais se fera sans problème.

\section{CONTRIBUTIONS DES AUTEURS}

Dans la réalisation de la présente étude, GXK, l'investigateur principal et J-MD ont élaboré le protocole de recherche, dirigé et encadré cette étude dans la collecte et le traitement des données ils ont aussi rédigé le manuscrit ; RKFMA et ITI ont participé à l'encadrement scientifique et correction dudit manuscrit.

\section{REMERCIEMENTS}

L'équipe de chercheurs adresse ces sincères remerciements au Ministère de l'Enseignement Supérieur et de la Recherche Scientifique du Bénin pour l'initiative «Appui aux doctorants » dont ils ont bénéficié. Nous saisissons également l'opportunité pour remercier Dr Daouda Orou BELLO du Laboratoire des Sciences du Sol et Dr Léa AITONDJI du Laboratoire d'Ecologie Appliquée pour leur contribution particulière apportée à cet article. Nos sincères remerciements aux évaluateurs pour leur importante contribution dans l'amélioration de la qualité du manuscrit. 


\section{REFERENCES}

Albrecht A, Kandji ST. 2003. Carbon sequestration in tropical agroforestry systems. Agriculture, Ecosystems and Environment, 99: 15-27. DOI : http://dx.doi.org/10.1016/S01678809(03)00138-5

Arrouays D, Balesdent J, Germon JC, Jayet PA, Soussana JF et Stengel P. 2002. Contribution à la lutte contre l'effet de serre. Stocker du carbone dans les sols agricoles de France? Expertise scientifique collective. Synthèse du rapport. INRA (France), $32 \mathrm{pp}$.

Bello OD, Saïdou A, Ahoton EL, Ezin AV, Avaligbé JFY, Akponikpè PBI, and Aho N. 2017. Assessment of organic carbon stock in cashew plantations (Anacardium occidentale L.) in Benin (West Africa). International Journal of Agriculture and Environmental Research, 3(4): 34713495.

Boer GJ, Flato G, Ramsden D. 2000. A transient climate change simulation with greenhouse gas and aerosol forcing: projected climate for the 21th century. Clim. Dyn., 16: 427-450.

Boulmane M, Regina IS, Khia A, Oubrahim H. 2013. Estimation du stock de carbone dans les iliçaies du Haut Atlas Marocain. Ann. Rech. For. Maroc, 20(2): 275-286.

Diatta AA, Ndour N, Manga A, Sambou B, Faye CS, Diatta L, Goudiaby A, Mbow C, Dieng SD. 2016. Services écosystémiques du parc agroforestier à Cordyla pinnata (Lepr. ex A. Rich.) Milne-Redh. dans le Sud du Bassin Arachidier (Sénégal). Int. J. Biol. Chem. Sci., 10(6): 2511-2525.

Djègo J, Sinsin B. 2006. Impact des espèces exotiques plantées sur la diversité spécifique des phytocénoses de leur sous-bois. Syst. Geog. Pl, 76: 191-209.

Djègo JGM. 2006. Phytosociologie de la végétation de sous-bois et impact écologique des plantations forestières sur la diversité floristique au Sud et au
Centre du Bénin. Thèse de Doctorat, Université d'Abomey-Calavi, 388 p.

Durot C. 2013. Evaluation et comparaison des stocks de carbone des systèmes agroforestiers à base de cacaoyers du Centre Cameroun : Cas de l'arrondissement de Bokito. Mémoire d'Ingénieur agricole, Institut Supérieur d'Agriculture de Lille.83 p.

FAO. 1997. State of the world's forest. World end publication, Oxford, UK, 1-5 pp.

FAO. 2002. La séquestration du carbone dans le sol pour une meilleure gestion des terres. Rapport sur les ressources en sols du monde 96, Rome, 70 p.

FAO. 2012. Situation des plantations du monde, Rome. $66 \mathrm{p}$

Frédérick D. 2015. Je suis Charlie Je suis Paris 2015 Des antidotes au chaos du monde? Limours, les éditions Héliomir. $152 \mathrm{p}$.

Fissore C, Giardina CP, Kolka RK, Trettin CC, King GM, Jurgensen MF, Barton CD, McDowell S. Douglas. 2008. Temperature and vegetation effects on soil organic carbon quality along a forested mean annual temperature gradient in North America. Global Change Biology, 14: 193-205. https://slideplayer.fr/slide/1319344/

GIEC. 2013. Résumé à l'intention des décideurs, Changements climatiques 2013: Les éléments scientifiques. Contribution du Groupe de travail I au cinquième Rapport d'évaluation du Groupe d'experts intergouvernemental sur l'évolution du climat. Cambridge, Royaume-Uni et New York : Cambridge University Press. https://www.ipcc.ch/pdf/assessmentreport/ar5/wg1/WG1AR5_SummaryVol ume_FINAL_FRENCH.pdf

IPCC (Intergovernmental Panel on Climate Change). 2003. Good Practice Guidance for Land Use, Land-use Change and Forestry (LULUCF). Institute for Global Environmental Strategies, Hayama, 
Japan.

https://www.ipccnggip.iges.or.jp/public/gpglulucf/gpglulu cf_files/GPG_LULUCF_FULL.pdf

IPCC. 2007. Climate Change 2007: The Physical Science Basis. Contribution of Working Group I to the Fourth Assessment Report of the Intergovernmental Panel on Climate Change. Cambridge University Press, Cambridge, United Kingdom and New York, NY, USA, 996 p.

MacDiken KG. 1997. A guide to monitoring carbon storage in forestry and agroforestry projects. Forest Carbon Monitoring Program. Winrock Internation Institute for Agricultural Development, Arlington, USA, $91 \mathrm{p}$.

Mbow C, Verstraete MM, Sambou B, Diaw AT, Neufeldt H. 2014. Allometric maize) en zone Soudanienne du Bénin. Int. J. Biol. Chem. Sci., 6(5): 2066-2082.

Medlyn B, De Kauwe M. 2013. Carbon dioxide and Water use in forests. Research News. https://www.researchgate.net/publication /285794139_Carbon_dioxide_and_water _use_in_forests_research_news_and_vie Ws.

MEPN. 2010. Plan d'Aménagement Participatif de la Forêt Classée de Pahou, Ministère de l'Environnement, de l'Habitat et de l'Urbanisme, 158 p.

Montagnini F, Nair P. 2004. Carbon sequestration: An underexploited environmental benefit of agroforestry systems. Agroforestry Systems, (61): 281-295.

http://www.scirp.org/(S(1z5mqp453edsn p55rrgjct55))/reference/ReferencesPaper s.aspx?ReferenceID=2111408.

Mouwembe MAB, Ifo SA, Binsangou Malonga S, Koubouana F. 2017. Variabilité spatiale des stocks de carbone organique du sol des savanes dans les réserves de Lésio-Louna et de Léfini, plateaux Tékés, République du Congo.
Afrique SCIENCE, 13(3): 297 - 307. http://www.afriquescience.info

Muoghalu IJ. 2014. Vulnérabilité des systèmes biophysiques et socioéconomiques des savanes et formations boisees d'Afrique occidentale et centrale au changement climatique. African Forest Forum, 2(14): 35 p. https://www.tandfonline.com/doi/full/10. 1080/10549811.2017.1296776?af=R

Ngueguim JR, Zapfack L, Noumi VN, Onana D, Betti JL, Riera B. 2015. Expériences sylvicoles au Cameroun : Croissance, mortalité et adaptabilité des espèces de bois d'oeuvre dans la station forestière de Mangombé (1964-2010). Int. J. Biol. Chem. Sci., 9(6): 2789-2807. DOI : http://dxdoi.org/10.4314/ijbcs.v9i6.22.

Palm CA, Woomer PL, Alegre J, Arevalo L, Castilla C, Cordeiro DG, Feigl B, Hairiah K, Kotto-Same J, Mendes A, Moukam A, Murdiyarso D, Njomgang R,Parton WJ, Ricse A, Rodrigues V, Sitompul, SM, Van Noordwijk M. 2000. Carbon séquestration and trace gas émissions in slash-and-burn and alternative landuses in, the humid tropics. Final Report, Alternatives to Slash and Burn (ABS). Climate Change Working Group, Phase II. ICRAF, Nairobi, Kenya. 29 p.

Pascal C, Peter S. 2015. Climat: 30 questions pour comprendre la Conférence de Paris, Éditions Les Petits Matins. 200 p.

Rondeux J. 1999. Les Mesures des Arbres et des Peuplements Forestiers. Les Presses Agronomiques de Gembloux : Gembloux, Belgique; $522 \mathrm{p}$.

Reichstein M. 2007. Impacts of Climate Change on Forest Soil Carbon: Principles, Factors, Models, Uncertainties. Forestry and Climate Change. $\mathrm{CAB}$ International: Wallingford, UK; 127-135.

Saïdou A, Dossa AFE, Gnanglè PC, Balogoun I, Aho N. 2012. Evaluation du stock de carbone dans les systèmes agroforestiers 
à karité (Vitellaria paradoxa C.F. Gaertn.) et à néré (Parkia biglobosa Jacq. G. Don) en zone Soudanienne du Bénin. Bulletin de la Recherche Agronomique $\mathrm{du}$ Bénin (BRAB). 9 p. http://www.slire.net/document/1737

Thompson ID, Baker JA et Ter-Mikaelian M. 2003. A review of the long-term effects of post-harvest silviculture on vertebrate wildlife, and predictive models, with an emphasis on boreal forests in Ontario, Canada. Forest Ecology and Management, 177(1-3): 441-469. https://cfs.nrcan.gc.ca/publications?id=2 1497

Valentini 2007. Evaluation de la séquestration de carbone dans des plantations agroforestières et des jachères issues d'une agriculture migratoire dans les territoires autochtones de Talamanca au Costa ; Mémoire présenté à la Faculté des études supérieures de l'Université Laval dans le cadre du programme de maitrise en biologie végétale pour l'obtention du grade de Maître es sciences (M.Sc) ; Faculté des Sciences de l'Agriculture et de l'alimentation,
Université Laval Québec. https://corpus.ulaval.ca/jspui/bitstream/2 0.500.11794/18894/1/24293.pdf

Volkoff B, Willaime P. 1976. Notice explicative $n^{\circ}$ 66. Carte pédologique de reconnaissance de la République populaire du Bénin à 1/200 000. Feuille de Porto- Novo (1). Paris, France, ORSTOM, $39 \quad$ p. http://horizon.documentation.ird.fr/exldoc/pleins_textes/pleins_textes_6/b_fdi_ 43-44/010005694.pdf.

Vroh BTA, Tiebre MS, N'guessan KE. 2014. Diversité végétale urbaine et estimation du stock de carbone: cas de la commune du Plateau Abidjan, Côte d'Ivoire. Afrique Science, 10(3): 329-340. https://www.ajol.info/index.php/afsci/art icle/view/109751

Yameogo G, Yelemou B, Boussim IJ, Traore D. 2013. Gestion du parc agroforestier du terroir de Vipalogo (Burkina Faso): contribution des ligneux à la satisfaction des besoins des populations. Int. J. Biol. Chem. Sci., 7(3): 1087-1105. DOI : http://dx.doi.org/10.4314/ijbcs.v7i3.16 